Trauma Berufskrankh 2007 - 9[Suppl 3]:

S390-S396

DOI 10.1007/s10039-007-1243-2

Online publiziert: 28. Juni 2007

(c) Springer Medizin Verlag 2007
D. Seybold · J. Geßmann · L. Özokyay · G. Muhr · M. Graf Chirurgische Klinik und Poliklinik, BG-Kliniken Bergmannsheil, Ruhr-Universität Bochum, Bochum
Posttraumatische Fehlstellungen mit großen Achsfehlstellungen sind aufgrund einer verbesserten Primärversorgung insgesamt selten geworden. Die Schwierigkeit bei sehr großen Fehlstellungen liegt

1. in der Korrektur der knöchernen Fehlstellung an sich und

2. in der Korrektur der Weichteile, die sich der Deformität über einen längeren Zeitraum angepasst haben.

Große Fehlstellungen sind meist bei älteren Patienten mit zusätzlich schlechter Knochenqualität und Durchblutungssituation anzutreffen. Dies erfordert eine weichteilschonende und somit graduelle Korrektur.

Korrekturverfahren sind die akuten und graduellen Techniken sowie die internen, externen und kombinierten Osteosyntheseverfahren. Die akute Korrektur mit interner Osteosynthese hat für den Patienten den höchsten Komfort und die kürzeste Behandlungszeit. Voraussetzung ist eine gute Knochen- und Weichteilsituation. Bei großen Fehlstellungen sind die Weichteilverhältnisse jedoch in der Regel schlecht, und es bedarf einer weichteilschonenden Korrektur und Osteosynthese über einen Fixateur. Der Ringfixateur bietet die Möglichkeit der weichteilschonenden Knochenfixation; sehr große Fehlstellungen können kontinuierlich unter Vollbelastung korrigiert werden. Der Patientenkomfort ist jedoch aufgrund der aufwändigen Fixateurmontage und der langen Behandlungszeit gering.
Im Weiteren werden die einzelnen Korrekturverfahren bei sehr großen Fehlstellungen dargestellt.

\section{Akute Korrektur}

Bei Fehlstellungen mit großem Angulationsausmaß führt sie in Abhängigkeit von ihrer Entfernung zur Fehlstellung auf der konvexen Seite der Achsabweichung zu einem Weichteilüberschuss, auf der konkaven Seite zu einer Weichteildehnung [5]. Dies bedingt, dass je nach Richtung der Fehlstellung und der Weichteilsituation unterschiedlich große Korrekturen akut durchgeführt werden können. Eine Valgusfehlstellung des Oberschenkels kann meist problemlos akut korrigiert werden, da es zu einer Weichteilentlastung auf der medialen Seite des Oberschenkels kommt und somit keine Gefahr einer Nerven-Gefäß-Verletzung besteht. Hingegen sind die akuten Korrekturen von Valgus- und Außenrotationsfehlstellungen der proximalen Tibia nur mit gleichzeitiger vollständiger Neurolyse des N. peronaeus möglich. Letztere beinhaltet nicht nur die Freilegung des Nervs, sondern auch die vollständige Durchtrennung der muskulären Septen der Peronäalmuskulatur. In $\square$ Tab. 1 sind die günstigen Richtungen für akute Korrekturen der unteren Extremität angegeben.

\section{Plattenosteosynthese}

Die akute Korrektur über eine interne Plattenosteosynthese ist bei sehr ausgeprägten Fehlstellungen nur bei guter Weichteilsitu- ation zu empfehlen. Die gerade oder anatomisch vorgeformte Platte dient dann als Repositions- und Retentionsinstrument. Nach einer schließenden oder neutralen Osteotomie kommt es aufgrund der hohen Weichteilspannung nicht automatisch zu einer Ausrichtung der Fehlstellung. Diese muss gewissermaßen an die Platte reponiert werden, um eine achsgerechte Korrektur zu erzielen ( Abb. 1).

\section{Kirschner-Draht-Osteosynthese}

Mit ihr können v. a. im Bereich der kleinen Knochen bei Fuß- oder kindlichen Fehlstellungskorrekturen die Korrekturergebnisse nach Osteotomieschluss fixiert werden (ब Abb. 2). Der größte Vorteil bei der Verwendung von Drähten ist die Möglichkeit, diese perkutan mit geringem Weichteiltrauma einbringen und sie nach Konsolidierung der Korrekturosteotomie ambulant entfernen zu können. Eine ausgiebige, denudierende Weichteilpräparation, wie es bei internen Plattenosteosynthesen häufig erforderlich ist, entfällt. Die Kirschner-Draht-Osteosynthese muss jedoch durch einen Gipsverband geschützt werden und ist nicht belastungsfähig.

\section{Nagelkorrektur}

Korrekturen über intramedulläre Kraftträger setzen einen intakten Markraum voraus. Um über nur eine Osteotomie korrigieren zu können, muss die Fehlstellung in einem sehr kurzen Knochensegment lokalisiert sein. Bei langbogigen Fehlstellungen 


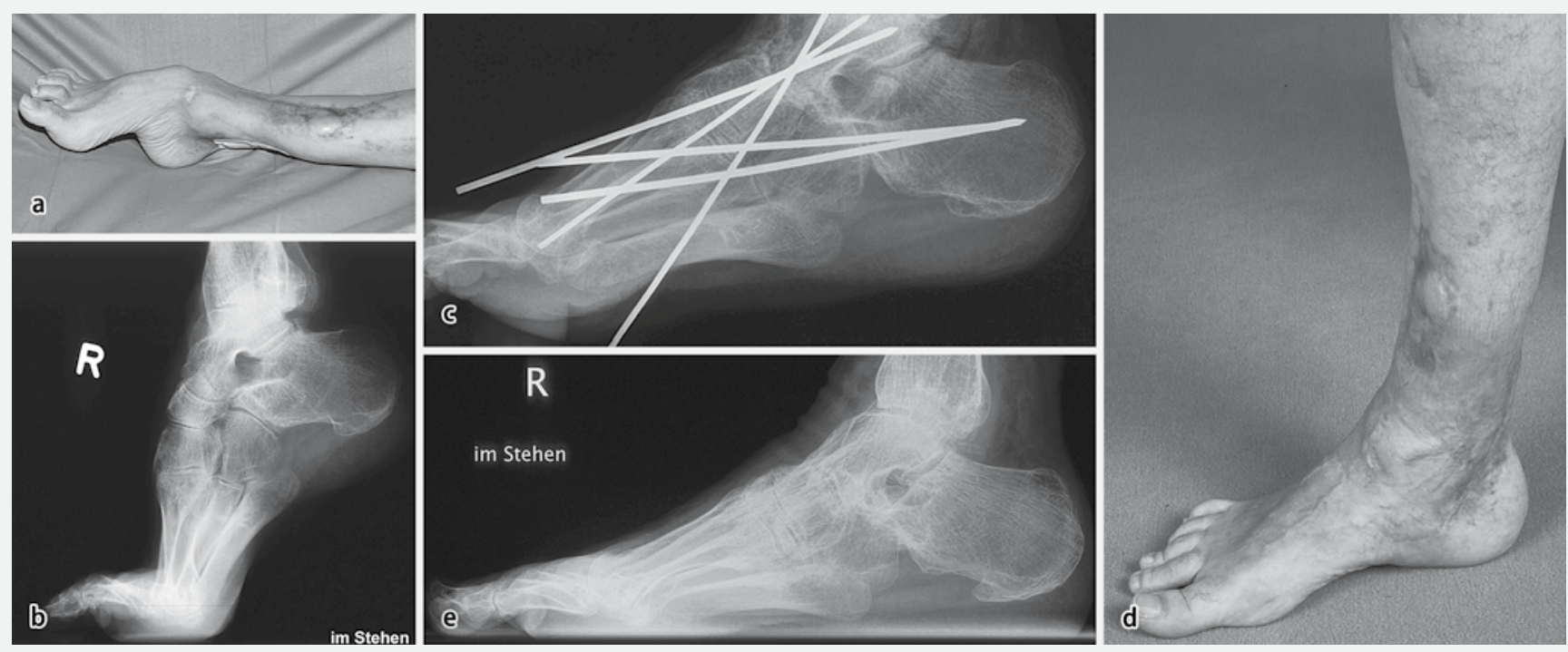

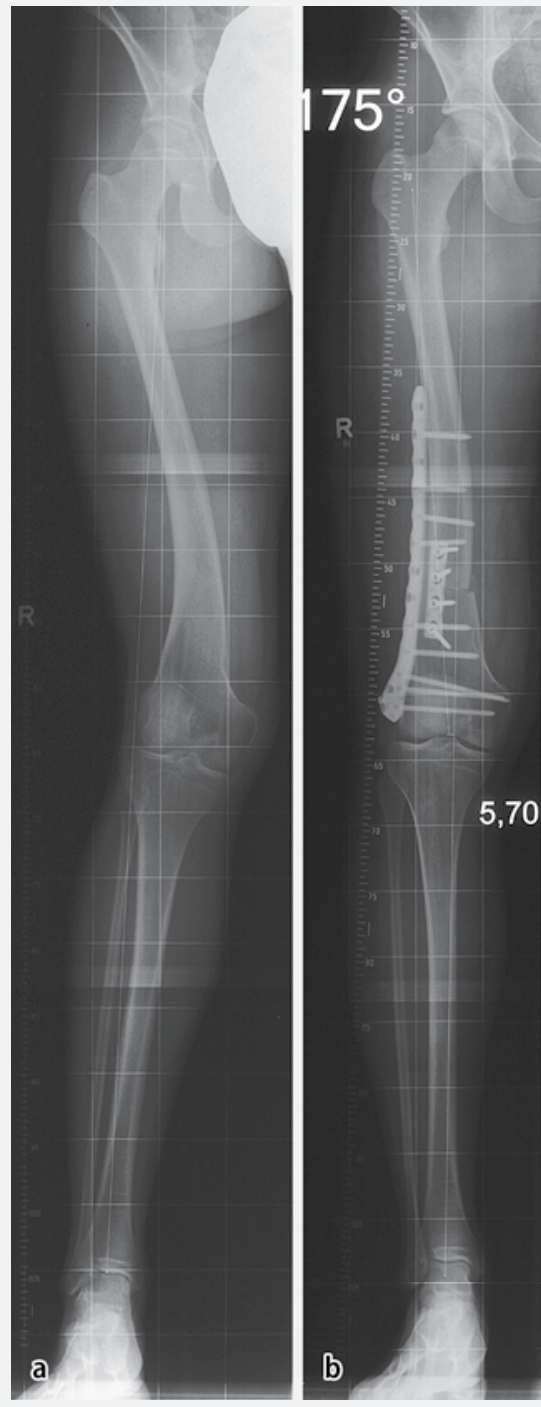

Abb. $1 \Delta$ Akute Korrektur einer femoralen Valgusfehlstellung von $30^{\circ}$ über eine supramalleoläre Osteotomie mit winkelstabiler Plattenosteosynthese, mechanische Beinachse bei nicht vollständiger Korrektur der Gelenkwinkel vollständig korrigiert
Abb. $2 \Delta$ Posttraumatische Spitzfußstellung, Akutkorrektur über eine Closing-Wedge-Osteotomie der Fußwurzel mit Kirschner-Draht-Osteosynthese

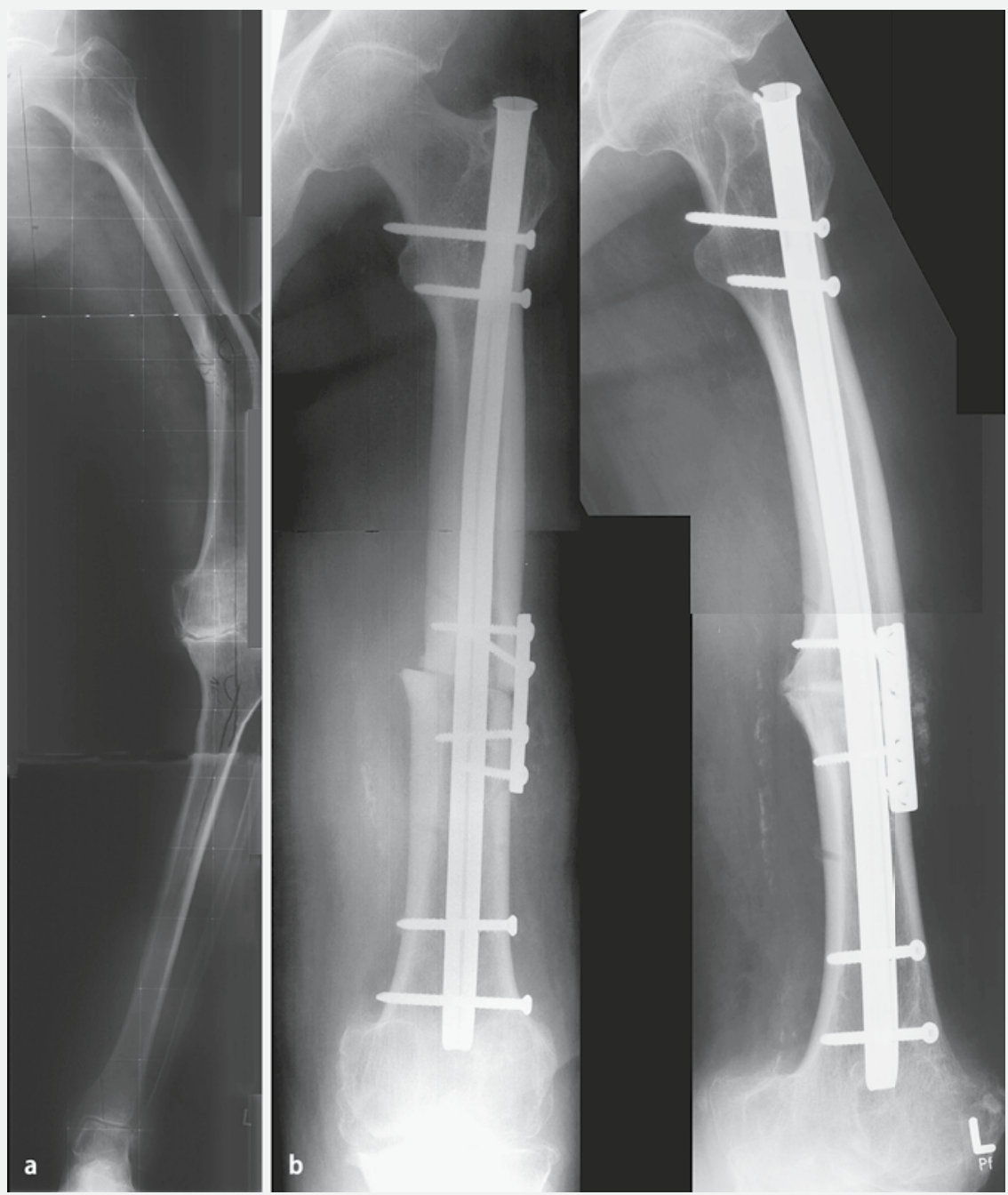

Abb. $3 \Delta$ Kurzbogige diaphysäre Varusfehlstellung des Femurs, akute Korrektur über eine verkürzende Closing-Wedge-Osteotomie mit Nagelosteosynthese und Antirotationsplatte 


\section{Sondersituationen}
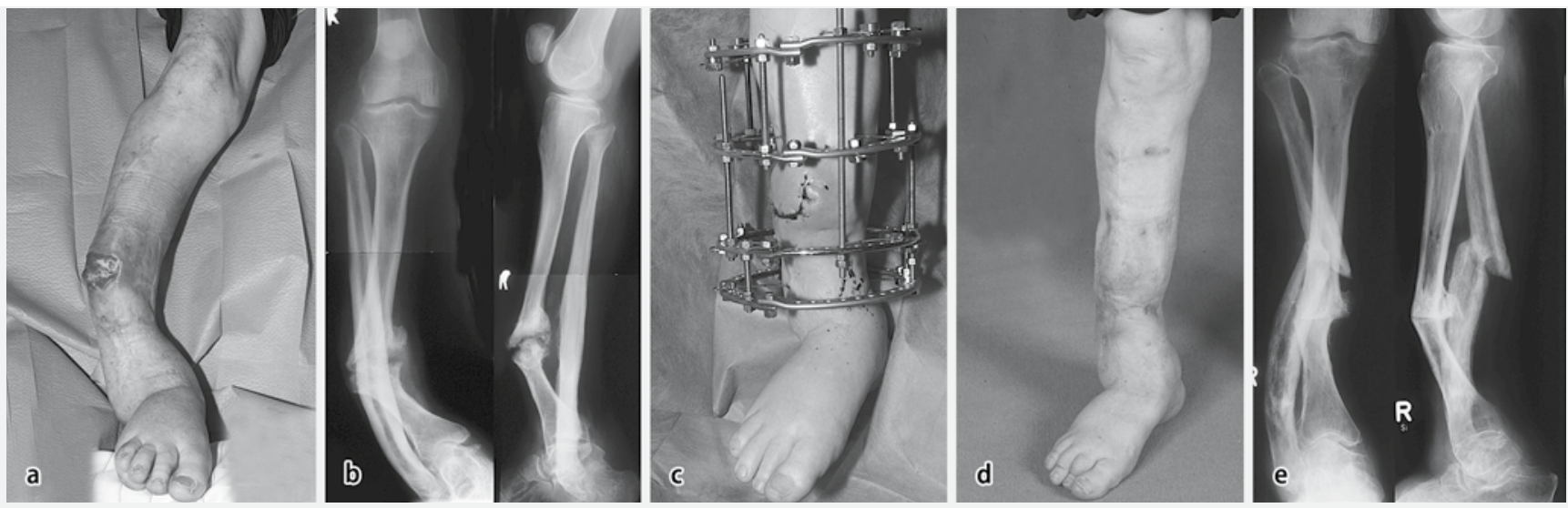

Abb. $4 \Delta$ Septische Tibiapseudarthrose nach Kriegsverletzung mit hypertrophierter Fibula, Resektion der Pseudarthrose und akute Korrektur der Fehlstellung mit Standard-Ilisarov-Ringfixateur

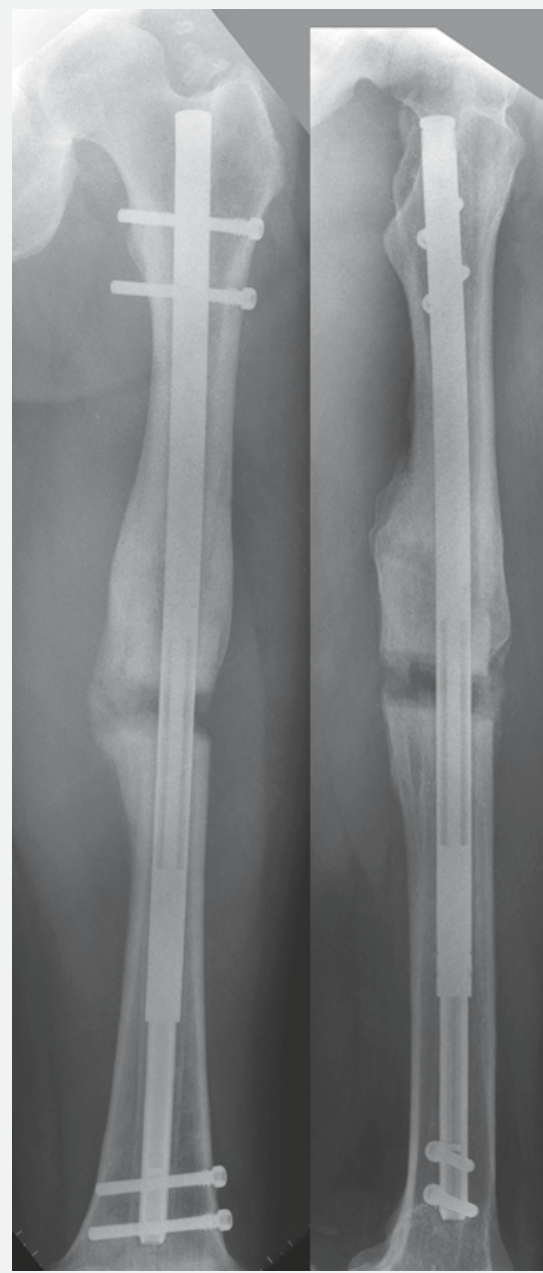

sind Etagenosteotomien erforderlich, um eine für den Nagel gerade medulläre Ausrichtung zu erhalten. Diese haben jedoch den Nachteil einer hohen Pseudarthrosenrate, da sowohl die intramedulläre Durchblutung durch den Nagel als auch die periostale Durchblutung durch die Osteotomie kompromittiert werden.
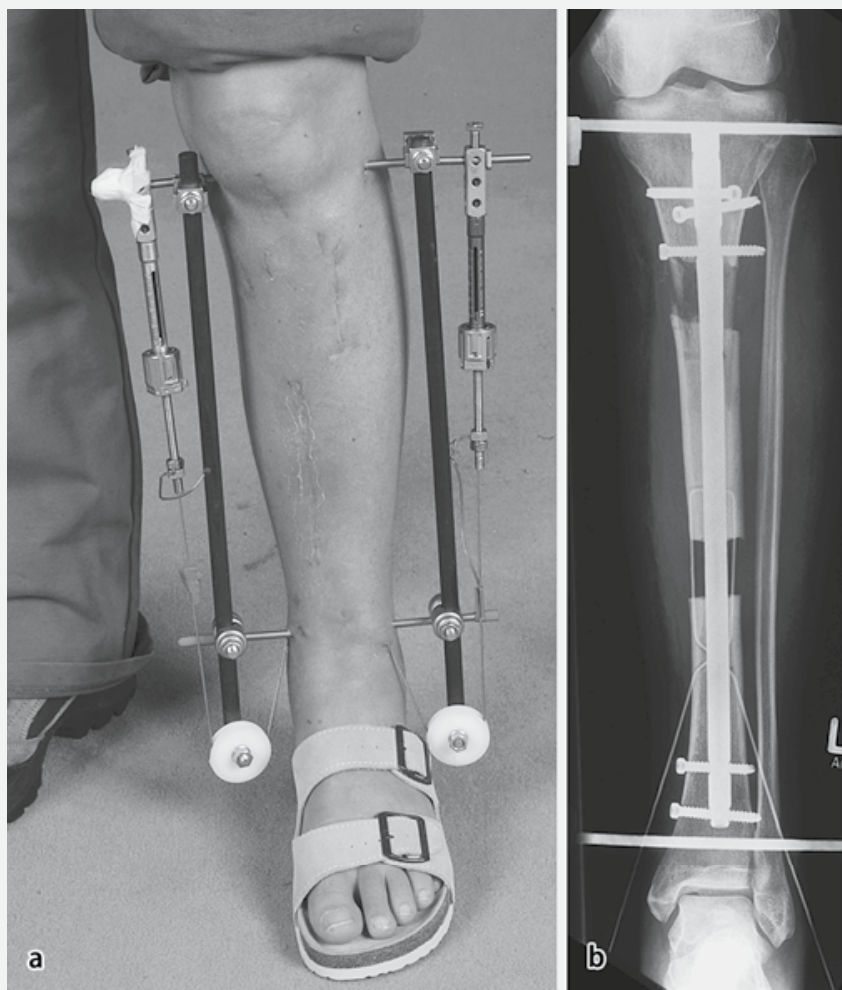

Abb. $6 \Delta$ Tibiasegmenttransport über einen Seilzug mit intramedullärer Nagelosteosynthese

Abb. 54 Femurverlängerung mit intramedullärem Distraktor (ISKD)

Die Korrektur über einen intramedullären Kraftträger eignet sich insbesondere bei kurzen diaphysären Angulationsund Rotationsfehlstellungen. Durch das Einbringen des Nagels kommt es zu einer selbstständigen Ausrichtung der Knochenachse. Bei metaphysär gelegenen Fehlstellungen ist die Korrektur von Angulations- fehlstellungen über den richtigen Eintrittspunkt des Nagels zu erreichen. Dieser bereitet jedoch häufig Schwierigkeiten, da der kurze metaphysäre Gelenkanteil mit der Nagelverriegelung nicht immer stabil fixiert werden kann. Wenn ausgeprägte Fehlstellungen aufgrund der Weichteilspannung nicht längenneutral akut korrigiert werden 


\begin{tabular}{|c|c|c|c|c|c|}
\hline Oberschenkel & + & - & Unterschenkel & + & - \\
\hline \multirow[t]{3}{*}{ Proximal } & Varus & Valgus & \multirow[t]{3}{*}{ Proximal } & Valgus & Varus \\
\hline & Flexion & Extension & & Flexion & Extension \\
\hline & Iro & Aro & & Aro & Iro \\
\hline \multirow[t]{3}{*}{ Distal } & Varus & Valgus & \multirow[t]{3}{*}{ Distal } & Varus & Valgus \\
\hline & Flexion & Extension & & Flexion & Extension \\
\hline & Aro & Iro & & Iro & Aro \\
\hline
\end{tabular}

\section{Infobox 1: Internetlinks}

- Analyse der Deformität: http://www.spatialcad.com

- Planung der Korrektur: http://www.spatialframe.com

können, bietet sich die Möglichkeit, durch eine Segmentresektion mit Verkürzung und ggf. späterer Verlängerung dennoch eine Akutkorrektur zu erreichen (• Abb. 3).

\section{Ringfixateur}

Er eignet sich bei schlechten Weichteilverhältnissen zur Knochenstabilisierung nach akuter Korrektur über eine Osteotomie oder Resektion einer Fehlstellungspseudarthrose (• Abb. 4) [3, 4, 8]. Die Osteosynthese mit dem Ringfixateur ist belastungsstabil und ermöglicht den $\mathrm{Pa}$ tienten nach abgeheilten Weichteilverhältnissen eine Vollbelastung. Die Stabilität des Ringsystems ist grundsätzliche Voraussetzung, um eine Knochenheilung nach Korrekturosteotomie erzielen zu können. Dies wird beim Ringfixateur durch die Verwendung von gekreuzten 1,8-mm-Olivendrähten und/oder 6-mmKnochenschrauben erzielt. Ein regelmäBiges Nachspannen ist unverzichtbar, um die Osteotomiekompression aufrechtzuerhalten.

\section{Kontinuierliche Korrekturen}

Mit diesen Verfahren können viel größere Fehlstellungsradien korrigiert werden als mit den akuten Maßnahmen [2, 5, 7]. Die Weichteile werden bei der kontinuierlichen Korrektur mit einer Distraktion von 0,5-2 mm am Tag sehr langsam an die auftretende Weichteilspannung angepasst. So ermöglicht die langsame Korrektur auch bei schlechten Weichteilsitu- ationen eine Behandlung großer Fehlstellungen ohne weichteilplastische Eingriffe. Das Risiko einer Schädigung von neurovaskulären Strukturen ist bei den kontinuierlichen Korrekturverfahren deutlich geringer als bei den akuten Verfahren. Der Transport kann beim Auftreten von Gefühls- oder Hautdurchblutungsstörungen sofort gestoppt oder zurückgesetzt werden.

Als Osteosyntheseverfahren kommen der Ringfixateur als Standard-IlisarovFixateur oder als Taylor-Spatial-Frame sowie unilaterale Fixateure zum Einsatz. Als intramedullärer Distraktor zur Längenkorrektur von Femur und Tibia stehen der ISKD-Nagel (• Abb.5) ebenso wie Kombinationen von intramedullärem Kraftträger und Fixateur externe zur Verfügung (• Abb. 6). Der Ringfixateur wird bei sehr ausgeprägten Fehlstellungen mit schlechten Weichteilen zur kontinuierlichen Korrektur bevorzugt. Er ist postoperativ voll belastungsfähig und bietet die Möglichkeit, kombinierte Fehlstellungen simultan zu korrigieren [7].

Der Taylor-Spatial-Frame als Weiterentwicklung des Standard-Ilisarov-Fixateurs wurde 1994 von J. C. Taylor in Memphis, USA, entwickelt [10]. Die Hexapodkinematik, wie sie bei Flugsimulatoren und im Maschinenbau eingesetzt wird, wurde auf den Standard-IlisarovFixateur übertragen. Die Hexapodanordnung von 6 verstellbaren Streben zwischen den zu korrigierenden Ringebenen ermöglicht eine simultane Korrektur von mehrdimensionalen Deformitäten in 6 Achsen des Raums [5, 9]. Diese Weiterentwicklung eröffnete in der primären und der posttraumatischen Deformitätenkorrektur wesentlich größere Einsatzmöglichkeiten bei gleichzeitig größerer Flexibilität. Ebenso entfallen aufwändige
Trauma Berufskrankh 2007 - 9[Suppl 3]: S390-S396

DOI 10.1007/s10039-007-1243-2

(c) Springer Medizin Verlag 2007

D. Seybold ·J. Geßmann · L. Özokyay .

G. Muhr · M. Graf

Korrekturverfahren bei

außergewöhnlichen

Fehlstellungen

\section{Zusammenfassung}

Fehlstellungskorrekturen bei posttraumatischer Weichteil- und Knochensituation mit großem Fehlstellungsausmaß erfordern eine schonende und meist graduelle Korrektur, um sekundäre Gefäß-, Nerven- oder Weichteilschäden zu vermeiden. Akutkorrekturen können nur bei guter Weichteildeckung und günstiger Korrekturrichtung durchgeführt werden. Der Ringfixateur und insbesondere der Taylor-Spatial-Frame eignen sich im Besonderen für die graduelle Korrektur von Fehlstellungen mit großem Fehlstellungsausmaß. Eine simultane Korrektur von mehrdimensionalen Fehlstellungen mit dem Taylor-Spatial-Frame ist ohne große Fixateurumbauten möglich. Die Patienten können sofort voll belasten und den Transport selbstständig durchführen.

\section{Schlüsselwörter}

Deformitätenkorrektur · Akutkorrektur · Ilisarov-Ringfixateur - Taylor-Spatial-Frame . Belastung

\section{Correction procedures in unusual types of malalignment}

\section{Abstract}

Severe posttraumatic deformities involving compromised soft tissue and bone demand gentle and usually gradual methods of correction so as to avoid secondary damage to the vascular system, nerves or soft tissue. Acute techniques are only possible when there is a good soft tissue mantle and the direction is safe. The llizarov ring fixator and the Taylor spatial frame are both known to be particularly suitable for gradual correction of severe deformities. The Taylor spatial frame allows correction of severe multiplanar deformities with no need for elaborate adaptations to it. The patients are capable of full weightbearing immediately and can be followed up as out-patients.

\section{Keywords}

Deformity correction - Acute correction . Ilizarov ring fixator - Taylor spatial frame . Weightbearing 

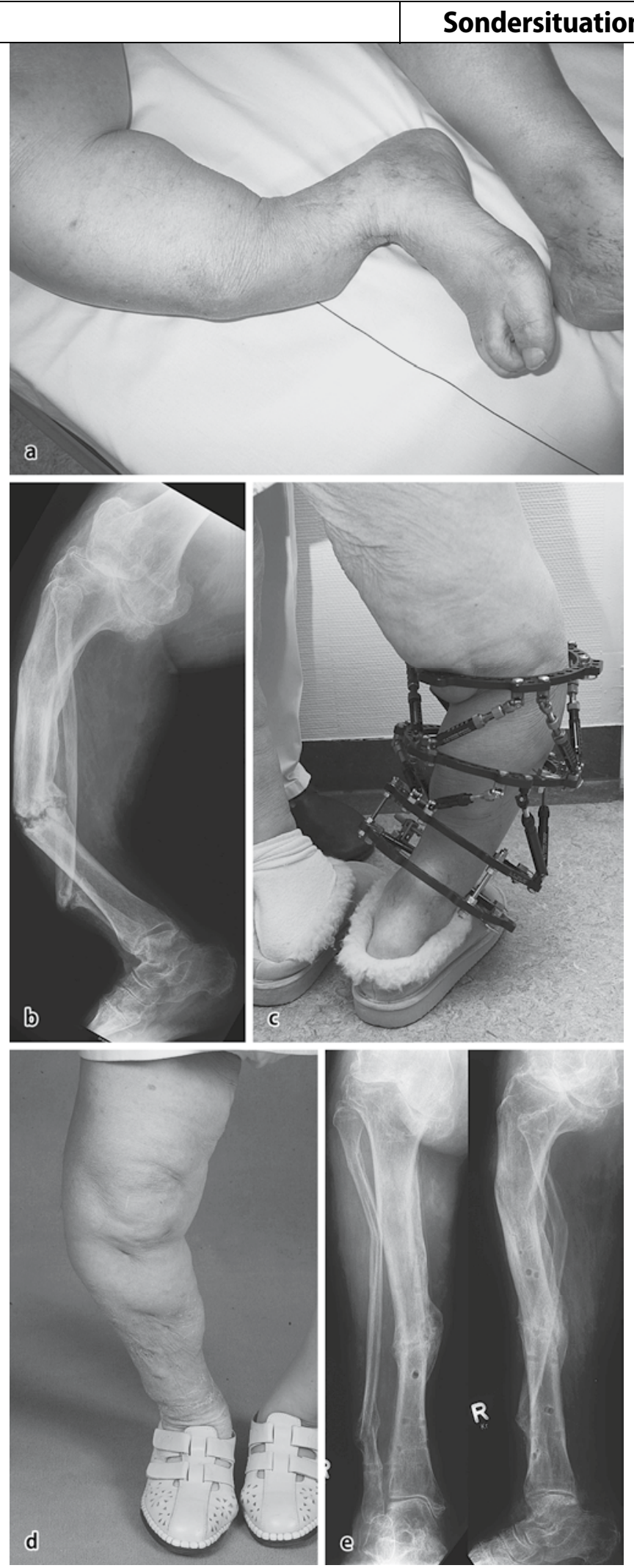

Abb. $7<$ Oligotrophe Unterschenkelpseudarthrose mit ausgeprägter Antekurvationsfehlstellung und Lateral-Thrust-Gonarthrose, geschlossene Korrektur mit TSF ohne additive Spongiosaplastik und z. T. unangenehme Fixateurum- und -anbauten. Sowohl die Analyse der Deformität als auch die Planung der Korrektur erfolgen bei der Anwendung des Taylor-Spatial-Frame (TSF) webbasiert

(- Infobox 1). Die Korrekturmodi und rungen ohne große Fixateurumbauten durchgeführt werden.

Im Weiteren werden die Anwendungsmöglichkeiten des Taylor-Spatial-FrameFixateurs in der posttraumatischen Deformitätenkorrektur von ausgeprägten Fehlstellungen der unteren Extremität dargestellt.

Die Montage des TSF erfolgt intraoperativ mit proximaler oder distaler Referenzierung, wobei der Referenzring als erster und wichtigster Ring zur späteren Korrektur streng orthogonal zur Knochenachse angebracht wird. Ein Vorbauen des Fixateurs ist nicht erforderlich und teilweise sogar hinderlich, da der Referenzring dann schwieriger auszurichten ist. Die Planung der TSF-Korrektur erfolgt mit dem webbasierten Programm http:// www.spatialframe.com im Total Residual Modus.

Intraoperativ bietet es sich an, FastFxStreben zu verwenden, um eine höhere Flexibilität in der Ringanordnung und ausrichtung zu haben, die postoperativ dann in Standardstreben ausgetauscht werden können. Nach Korrekturabschluss werden die Streben bei parallel stehenden Ringebenen mit Gewindestangen ausgewechselt, bei schief stehenden Ringebenen werden sie belassen und zusätzlich mit Gewindestangen die Ringposition fixiert. Bei Ringmontagen mit einer Rückfußtransfixation empfiehlt es sich, einen Verbandschuh mit Abrollsohle anzupassen, um eine Belastung der Extremität zu erleichtern. Die Korrektur der Fehlstellung können die Patienten selbstständig mit einem ausgehändigten Korrekturplan ambulant durchführen und müssen sich nur zur Kontrolle des Transports und für Strebenwechsel regelmäßig vorstellen.

\section{Unterschenkelkorrekturen}

Die Korrektur von ausgeprägten hypertrophen bzw. oligotrophen Fehlstellungspseudarthrosen des Unterschenkels mit dem TSF kann durch eine geschlossene Anlage einer stabilen 4-Ring-Montage erfolgen (• Abb. 7) $[1,8]$. Ein offenes Dekortizieren oder Augmentieren durch autologes oder allogenes Knochenmaterial ist bei hypertrophen und oligotrophen Fehlstellungspseudarthrosen nicht erforderlich. Bei intaktem Wadenbein ist regel- 
haft eine Wadenbeinosteotomie entsprechend der Korrekturrichtung durchzuführen. Durch eine kontinuierliche Distraktion in der Pseudarthrose mit einer Distraktionsgeschwindigkeit von 1-2 mm am Tag werden einerseits die Fehlstellung korrigiert und andererseits ein Distraktionsreiz auf die Pseudarthrose ausgeübt, der eine osteogene Stimulation bewirkt. Nach der Korrektur muss durch regelmäßiges Nachspannen des Fixateurs eine kontinuierliche Kompression bis zur Konsolidierung erfolgen. Die Fixateurtragezeit ist bei der Korrektur von ausgeprägten Fehlstellungspseudarthrosen sehr lange und beträgt im Durchschnitt 20 Wochen.

\section{Supramalleoläre Rückfußkorrekturen}

Das Zentrum der Fehlstellung nach traumatischen Fugenverletzungen liegt typischerweise intraartikulär bzw. gelenknah [11]. Die für die Korrektur erforderliche Osteotomie kann daher nicht auf Höhe der Fehlstellung erfolgen, sondern muss alternativ platziert werden. Eine Korrektur der Fehlstellung in der entfernten Osteotomie führt jedoch entsprechend der Osteotomieregel III zu einer anatomischen und mechanischen Fehlstellung [6]. Der TSF ermöglicht eine freie Platzierung des Korrekturzentrums (Origin) auch außerhalb des Fixateurarbeitsraums, sodass eine vollständige Korrektur der intraartikulären bzw. gelenknahen Fehlstellungen möglich ist.

Nach Fixateuranlage entsprechend der Rückfußfehlstellung erfolgt die supramalleoläre Osteotomie unter Schonung des Periosts als Bohrloch- oder perkutane Gigli-Sägen-Osteotomie (• Abb. 8). Die Osteotomie der Fibula sollte nicht auf gleicher Höhe wie die Tibiaosteotomie erfolgen, um nicht zu viel Instabilität im Bereich der Korrektur zu erhalten. Die Osteotomierichtung der Tibia orientiert sich an der Gelenkfläche und der Korrekturrichtung, die der Fibula nur an der Korrekturrichtung. Der Transport wird je nach Alter des Patienten und der Knochen- bzw. Weichteilsituation 5-12 Tage nach der Osteotomie mit einer Geschwindigkeit von $0,5-1,5 \mathrm{~mm}$ am Tag begonnen. Durch radiologische Kontrollen können die Korrektur und die Kallus-
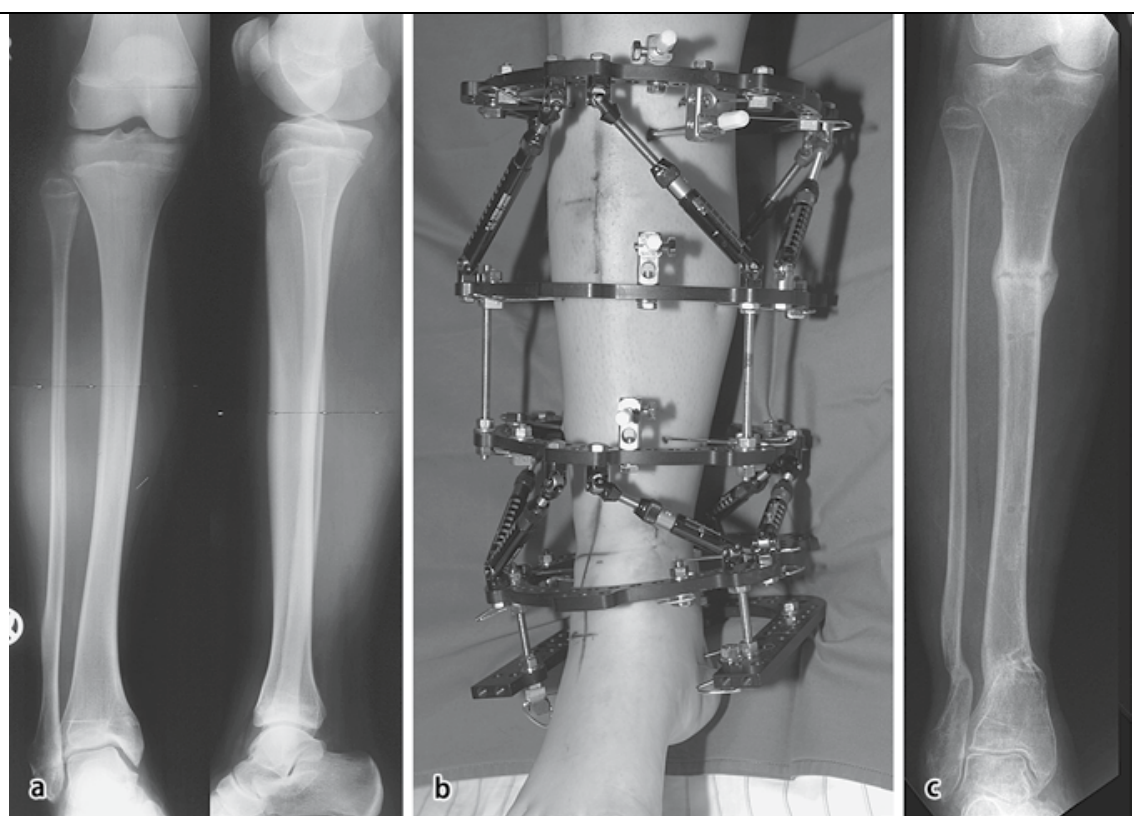

Abb. 8 \ Varusfehlstellung nach kindlicher Sprunggelenkfraktur mit sekundärer Kompensation und Verkürzung, Doppelosteotomie und Korrektur der Fehlstellung und des Längendefizits über eine Kallusdistraktion mit dem TSF

distraktion im Verlauf nachgehalten und ggf. korrigiert werden. Auf die Einnahme von NSAR und auf das Rauchen sollte während des Transports und der Konsolidationsphase generell verzichtet werden. Die Fixateurtragezeit variiert bei den Korrekturen über eine kurze Kallusdistraktionsstrecke sehr stark. Ein Healing-Index von 80 Tagen $/ \mathrm{cm}$ ist durchaus realistisch.

\section{Fazit für die Praxis}

Posttraumatische Fehlstellungen mit großem Fehlstellungsausmaß stellen hohe Anforderungen an das Korrekturverfahren und das verwendete Osteosynthesematerial. Die Weichteildurchblutung und Knochenvitalität sind posttraumatisch in der Regel beeinträchtigt und erfordern dadurch eine möglichst weichteil- und knochenschonende Korrektur. Dies gelingt mit externen Osteosynthesen und minimalinvasiven Osteotomien. Der Ringfixateur als Taylor-Spatial-Frame ist in der dreidimensionalen Korrektur von posttraumatischen Fehlstellungen gegenüber den internen Osteosynthesen und dem Standard-Ilisarov-Ringfixateur eine mittlerweile unverzichtbare Neuerung. Dreidimensionale Korrekturen können simultan und ohne große Fixateurumbauten durchgeführt wer- den. Dies ermöglicht es, nach Fixateuranlage und Osteotomie den Patienten ambulant zu führen und nur zur Kontrolle des Transports oder zum Strebenwechsel einzubestellen.

\section{Korrespondenzadresse}

\section{Dr. D. Seybold}

Chirurgische Klinik und Poliklinik, BG-Kliniken Bergmannsheil, Ruhr-Universität Bochum Bürkle-de-la-Camp-Platz 1, 44789 Bochum dseybold@mac.com

Interessenkonflikt. Der korrespondierende Auto gibt an, dass kein Interessenkonflikt besteht.

\section{Literatur}

1. Feldman DS, Shin SS, Madan S et al. (2003) Correction of tibial malunion and nonunion with six-axis analysis deformity correction using the Taylor spatial frame. J Orthop Trauma 17: 549-554

2. Feldman DS, Madan SS, Ruchelsman DE et al. (2006) Accuracy of correction of tibia vara: acute versus gradual correction. J Pediatr Orthop 26: 794-798

3. Möllenhoff G, Josten C, Muhr G (1997) Kallotaxis - Osteoneogenese durch Dehnung - eine schonende Möglichkeit der Beinlängenwiederherstellung nach posttraumatischer primärer Unterschenkelverkürzung? Zentralbl Chir 122: 970-973

4. Nho SJ, Helfet DL, Rozbruch SR (2006) Temporary intentional leg shortening and deformation to facilitate wound closure using the llizarov/Taylor spatial frame. J Orthop Trauma 20: 419-424

5. Paley D (2002) Six-axis deformity analysis and correction. In: Paley D (ed) Principles of deformity correction. Springer, Berlin Heidelberg New York 


\section{Sondersituationen}

6. Paley D, Herzenberg JE, Tetsworth K et al. (1994) Deformity planning for frontal and sagittal plane corrective osteotomies. Orthop Clin North Am 25: 425-465

7. Rodl R, Leidinger B, Bohm A et al. (2003) Correction of deformities with conventional and hexapod frames - comparison of methods. Z Orthop Ihre Grenzgeb 141: 92-98

8. Rozbruch SR, Helfet DL, Blyakher A (2002) Distraction of hypertrophic nonunion of tibia with deformity using llizarov/Taylor spatial frame. Report of two cases. Arch Orthop Trauma Surg 122: 295-298

9. Seide K (2003) Der Hexapodfixateur externe. Trauma Berufskrankh 5: 7-62

10. Taylor J (2006) Tibia shaft fractures: spatial frame. In: Wiss D (ed) Master techniques in orthopaedic surgery: fractures. Lippincott Williams \& Wilkins, Philadelphia

11. Verheyden P, Josten C (1998) Supramalleolar corrective osteotomy. Chirurg 69: 1178-1187 